\title{
Knockdown of Rhotekin 2 expression suppresses proliferation and induces apoptosis in colon cancer cells
}

\author{
XUEQIN PANG ${ }^{1 *}$, RUI LI ${ }^{1 *}$, DONGTAO SHI ${ }^{1}$, XUDONG PAN $^{2}$, CHEN MA $^{1}$, \\ GUANGBO ZHANG $^{1}$, CHUANYONG MU ${ }^{1,2}$ and WEICHANG CHEN ${ }^{1}$ \\ Departments of ${ }^{1}$ Gastroenterology and ${ }^{2}$ Respiratory, The First Affiliated Hospital \\ of Soochow University, Suzhou, Jiangsu 215006, P.R. China
}

Received July 28, 2017; Accepted September 22, 2017

DOI: 10.3892/ol.2017.7182

\begin{abstract}
Colon cancer is one of the most common malignant tumors in the human body, ranking second as a gastrointestinal tumor. It has a high incidence in Europe, America and China and more than 1 million new cases of colon cancer are reported worldwide each year. The incidence of colon cancer in China has increased from 12/0.1 million in the early 1970 s to $56 / 0.1$ million at present with an annual growth rate of $4.2 \%$, which far exceeds the international level (2\%). Rhotekin (RTKN) 2, a Rho-guanosine triphosphatase (GTPase) effector, has been reported to be anti-apoptotic. However, the molecular mechanism underlying the biological function of RTKN2 in colon cancer remains unknown. The present study investigated whether the mRNA expression level of RTKN2 was markedly higher in 30 human colon cancer specimens compared with adjacent non-cancerous tissues. The results showed that the protein expression level of RTKN2 was significantly higher in SW480 and HCT116 cells, compared with HIEC cells. Knockdown of RTKN2 in the SW480 and HCT116 colon cancer cells, by lentivirus-mediated RNA interference led to the notable inhibition of cell proliferation and cell cycle progression, by reducing the expression levels of the PCDA, Cyclin D1 and c-myc cell cycle-associated proteins. The inhibitory effect of RTKN2 silencing on the proliferation of colon cancer cells may be partially realized by inhibiting the Wnt/ $\beta$-catenin signaling pathway. Furthermore, the silencing of RTKN2 in the cells induced apoptosis by reducing the expression level of Bax and increasing the expression level
\end{abstract}

Correspondence to: Dr Chuanyong $\mathrm{Mu}$ or Dr Weichang Chen, Department of Gastroenterology, The First Affiliated Hospital of Soochow University, 188 Shizi Street, Suzhou, Jiangsu 215006, P.R. China

E-mail: muchuanyong@suda.edu.cn

E-mail: ccu18v@163.com

*Contributed equally

Key words: rhotekin 2, apoptosis, colon cancer cells, cell cycle, cell proliferation of $\mathrm{Bcl} 2$. These results show that RTKN2 is involved in the carcinogenesis and progression of human colon cancer, indicating that RTKN2 may be a molecular target in colon cancer therapy.

\section{Introduction}

Rapid economic development and changes in human life style, particularly the dietary structure, have resulted in an increase in the incidence of colon cancer. According to clinical data, colon cancer has become a global problem and ranks 3rd in all common malignant tumors in male and even 2 nd in female tumors. Thus it is a malignant tumor with a high incidence and is extremely difficult to treat (1). At present, the treatment for the disease is mainly comprehensive therapy combined with surgery, chemotherapy and other types of treatment. The therapeutic effect of early-stage colon cancer is relatively good and its 5-year survival rate is more than 90\%. However, the therapeutic effect on patients with middle- and advanced stage colon cancer is not satisfactory. Although early-stage colon cancer can be successfully found via colonoscopy and more patients can receive the self-healing treatment, more than $25 \%$ of patients have been accompanied with metastasis when diagnosed (2), and 20-45\% patients suffer from recurrence or distant metastasis after surgery (3). Therefore it is important to investigate the underlying mechanisms of the pathogenesis of colon cancer, not only for prevention and prognosis, but also for treatment.

Rhotekin (RTKN) 2, a novel Rho effector, is a member of the RTKN protein family. The two RTKN proteins, RTKN1 and 2, have homologues in the majority of animals and mammals, including horses, dogs, rats, and humans and each of the proteins has an N-terminal Rho-guanosine triphosphatase (GTPase) binding domain and pleckstrin homology domain (4). The similar protein architecture of RTKN proteins indicated that they may share functional characteristics (4). Previous studies showed that RTKN proteins regulate critical cell functions including cell proliferation and cell cycle progression, cytokinesis, apoptosis and transformation. Wei et al (5) reported the overexpression of RTKN2 in the majority of hepatocellular carcinoma (HCC) patients and demonstrated an association between RTKN2 expression and proliferation, apoptosis and metastatic progression. 
Table I. Primer sequences of genes.

\begin{tabular}{|c|c|c|c|}
\hline Gene & $\begin{array}{c}\text { Primer } \\
\text { sequence }\left(5^{\prime}-3^{\prime}\right)\end{array}$ & $\begin{array}{l}\text { Length } \\
\text { of amplification (bp) }\end{array}$ & $\begin{array}{c}\text { Annealing } \\
\text { temperature }\left({ }^{\circ} \mathrm{C}\right)\end{array}$ \\
\hline \multirow[t]{2}{*}{ RTKN2 } & F: ACAGTTCGCGTTGGAGATGGAG & 245 & 58 \\
\hline & R: GTCGAGCATTGCACACCATGAG & & \\
\hline \multirow[t]{2}{*}{$G A P D H$} & F: CACCCACTCCTCCACCTTTG & 218 & 58 \\
\hline & R: CCACCACCCTGTTGCTGTAG & & \\
\hline
\end{tabular}

RTKN2, Rhotekin 2; GAPDH, glyceraldehyde 3-phosphate dehydrogenase; F, forward; R, reverse.

Similarly, Liao et al (6) reported the anti-apoptotic effects of RTKN2 in bladder cancer cells. Furthermore, the overexpression of RTKN2 reduced viability and increased sensitivity to $25-\mathrm{OHC}(7)$, which is directly associated with apoptosis in hematopoietic and leukemic cells (8-10). Although the RTKN2 gene is associated with several cancer types, including HCC, bladder and breast cancer $(5,6,11)$, the expression pattern and biological functions of RTKN2 in human colon cancer have yet to be investigated.

In the present study, the role of RTKN2 in human colon cancer and the associated mechanisms were explored. Firstly, we found that the gene expression level of RTKN2 was markedly higher in human colon cancer tissues. Furthermore, we investigated the role of RTKN2, including cell proliferation, cell cycle and apoptosis in RTKN2 knockdown, as well as in SW480 and HCT116 colon cancer cells.

\section{Materials and methods}

Patients and tissue samples. Tumor tissues and paired non-cancerous tissues were collected from 30 patients with colon cancer who were admitted to the Department of Radiology, The First Affiliated Hospital of Soochow University (Suzhou, China) between 2010 and 2012. Ethics approval for the study was provided by the Independent Ethics Committee of The First Affiliated Hospital of Soochow University. Informed and written consent was obtained from all the patients or their advisers according to the Ethics Committee guidelines.

Cell lines. HIEC, SW480 and HCT116 cells were obtained from the Cell Bank of Shanghai Biology Institute, Chinese Academy of Science (Shanghai, China). Culture media were supplemented with $10 \%$ fetal bovine serum, $100 \mathrm{mg} / \mathrm{ml}$ penicillin $\mathrm{G}$ and $50 \mu \mathrm{g} / \mathrm{ml}$ streptomycin (Life Technologies; Thermo Fisher Scientific, Inc., Waltham, MA, USA). HIEC, SW480 and HCT116 cells were cultured in Dulbecco's modified Eagle's medium (DMEM; Life Technologies; Thermo Fisher Scientific, Inc.). The cells were maintained at $37^{\circ} \mathrm{C}$ in $5 \% \mathrm{CO}_{2}$.

Vector construction. pLKO.1, psPAX2 and pMD2.G were purchased from Addgene, Inc., (Cambridge, MA, USA). Three small hairpin RNAs (shRNAs; Generay Biotech Co., Ltd., Shanghai, China) targeting human RTKN2 mRNA were cloned into a lentiviral vector (PLKO.1). A non-specific scramble shRNA sequence (CCTAAGGTTAAGTCGCCCTCG) was used as a negative control. The constructs were then transfected into HIEC cells with lentiviral packaging vectors (psPAX2 and pMD2.G) using Lipofectamine ${ }^{\circledR} 2000$ (Invitrogen; Thermo Fisher Scientific, Inc.) according to the manufacturer's instructions. Viruses were collected $48 \mathrm{~h}$ subsequent to transfection and used to infect SW480 and HCT116 cells. After 48 h, the cells were processed for reverse transcription-quantitative polymerase chain reaction (RT-qPCR) and western blot analysis. The shRNA target sequence for RTKN2 was CAGGGAAAGAACAATAGAAGTCT (1967-1989).

$R N A$ extraction and RT-PCR. Total RNA was extracted using TRIzol and the purity and concentration of the extracted RNA were detected using a nucleic acid protein detector. The reverse transcription reaction system was in strict accordance with the instructions of the reverse transcription M-MLV first strand kit (Invitrogen; Thermo Fisher Scientific, Inc.). The fluorescence quantitative PCR of mRNA was performed according to the instructions of the fluorescence real-time quantitative PCR kit SYBR-Premix Ex Taq (Takara Biotechnology Co., Ltd., Dalian, China). The reaction parameters of RT-qPCR were: pre-denaturation at $94^{\circ} \mathrm{C}$ for $5 \mathrm{~min}$, denaturation at $95^{\circ} \mathrm{C}$ for $30 \mathrm{sec}$, annealing at $58^{\circ} \mathrm{C}$ for $30 \mathrm{sec}$, extension at $72^{\circ} \mathrm{C}$ for $30 \mathrm{sec}$, a total of 40 cycles, collection of fluorescence at $75-80^{\circ} \mathrm{C}$ and melting curve analysis at $65-95^{\circ} \mathrm{C}$ [with glyceraldehyde 3-phosphate dehydrogenase (GAPDH) as the internal reference]. The primers used for quantitative PCR were designed using Primer 5.0 software and synthesized by Invitrogen (Thermo Fisher Scientific, Inc.) after homologous alignment. The specific sequences, length of amplification and annealing temperature are shown in Table I.

Western blot analysis. Polyacrylamide electrophoresis was performed in a predetermined order and the loading amount of protein was $150 \mu \mathrm{g}$ per well. Electrophoresis was performed under $100 \mathrm{~V}$ when the markers began to be separated until all the markers were separated and the target bands were obtained. The protein was electronically transferred onto the polyvinylidene fluoride membrane under the $350 \mathrm{~mA}$ current for approximately $2 \mathrm{~h}$. The membrane was sealed using $5 \%$ bovine serum albumin/milk at room temperature for $1 \mathrm{~h}$, and the rabbit anti-human polyclonal antibody RTKN2 (dilution, 1:500; cat. no. PA5-25716; Invitrogen, Waltham, MA,USA), Rabbit anti-human monoclonal antibody (PCNA, $\beta$-catenin, Bax, Bcl-2, cyclin D1, c-myc and GAPDH; dilution, 1:1000; cat. nos. 13110, 8480, 5023, 4223, 2872, 13987 and 5174; Cell Signaling Technology, Inc., Danvers, MA, 
USA) were incubatedd at $4^{\circ} \mathrm{C}$ overnight. The goat anti-rabbit secondary polyclonal antibodies (dilution, 1:2,000; cat. no. 7074; Cell Signaling Technology, Inc.) were added for incubation at $37^{\circ} \mathrm{C}$ for $1 \mathrm{~h}$ on the second day. Finally, the exposure liquid was added, and images were captured using chemiluminescence apparatus.

Cell Counting kit (CCK)-8 assay. SW480 and HCT116 cells in the logarithmic growth phase were inoculated onto the 96-well plates and the cell density was adjusted to $2 \times 10^{3}$ and $200 \mu \mathrm{l}$ RPMI-1640 medium containing $10 \%$ fetal bovine serum was added, with 6 control wells for each group, prior to incubation for $24 \mathrm{~h}$. Then, $10 \mu \mathrm{l}$ CCK- 8 was added into each well for incubation in an incubator containing $\mathrm{CO}_{2}$ for $4 \mathrm{~h}$ and wells with phosphate-buffered saline (PBS) were used as controls. The absorbance (A) value at $450 \mathrm{~nm}$ was detected using the microplate reader (Thermo Fisher Scientific, Waltham, MA, USA). The growth curve was drawn with the mean (A) value as the ordinate and time as the abscissa.

Cell cycle assay. SW480 and HCT116 cells in the logarithmic growth phase were digested with trypsin (the digestion time should be as short as possible and the cells blown and beaten as slightly as possible), collected and washed twice with PBS to wash away the trypsin and surface impurities. The cells were incubated and stained using $10 \mu \mathrm{g} / \mathrm{ml}$ propidium iodide solution (PI) in $500 \mu \mathrm{l}$ PBS for $30 \mathrm{~min}$ (containing $100 \mu \mathrm{g} / \mathrm{ml}$ RNase). The cell cycle of chondrocytes treated in each group was analyzed using the Beckman flow cytometer, followed by statistical analysis using GraphPad software (GraphPad Software Inc., La Jolla, CA, USA).

Cell apoptosis assay. Adherent cells were digested with trypsin without ethylene diamine tetraacetic acid (the digestion time should be as short as possible to avoid false positives). The cells were washed with PBS twice (centrifuged for 5 min at $2,115 \times \mathrm{g}$ ) and $1-5 \times 10^{5}$ cells were collected, prior to the addition of $500 \mu \mathrm{l}$ binding buffer to suspend cells. Then, $5 \mu \mathrm{l}$ Annexin V-FITC and $5 \mu 1$ PI were added and mixed evenly for reaction for 5-15 $\mathrm{min}$ in the dark at room temperature. The cells were observed and detected using a flow cytometer (Thermo Fisher Scientific) within $1 \mathrm{~h}$ and the excitation wavelength $(E x=488 \mathrm{~nm})$ and emission wavelength $(E m=530 \mathrm{~nm})$ were measured. Green fluorescence of Annexin V was detected via the FITC channel (FL1) and PI red fluorescence was detected via FL3. Statistical analysis was performed using the GraphPad software (GraphPad Software Inc.).

Statistical analysis. Results were analyzed using the GraphPad Prism 5 software (GraphPad Software Inc.). The data were presented as mean \pm standard deviation $(\mathrm{SD})$. The independent sample t-test was used to compare the difference between the two groups, while analysis of variance was used for comparison of the multi-sample means. $\mathrm{P}<0.05$ was considered to indicate a statistically significant difference.

\section{Results}

RTKN2 is upregulated in human colon cancer tissues and cell lines. The mRNA expression levels of RTKN2 were compared between colon cancer tissues and adjacent non-cancerous tissues using RT-PCR. Overexpression of RTKN2 was confirmed in $90 \%(27 / 30)$ of the measured colon cancer tissues (Fig. 1A). The protein levels of RTKN2 were analyzed in six pairs of colon cancer tissues and matched adjacent normal tissues using western blot analysis. The results showed that the protein level of RTKN2 was markedly increased in colon cancer tissues, compared with the matched adjacent normal tissues (Fig. 1D and F). The present study also examined the mRNA and protein levels of RTKN2 in human colon cancer cell lines. The results demonstrated the mRNA and protein levels of RTKN2 were significantly higher in SW480 and HCT116 human colon cancer cell lines, compared with the HIEC cells (Fig. 1B, C and E).

RTKN2 is downregulated by siRNA in SW480 and HCT116 cell lines. To investigate the functions of RTKN2 in human colon cancer, we designed the shRNA targeting RTKN2 and packaged RTKN2 shRNA virus. The mRNA and protein expression levels of RTKN2 in response to the specific RTKN2 shRNA virus were then assessed using RT-PCR and western blot analysis, respectively. The results showed that the shRNA virus targering RTKN2 was able to efficiently inhibit endogenous RTKN2 in SW480 and HCT116 cells (Fig. 2A-C). Therefore, the RTKN2 shRNA virus was stably infections into SW480 and HCT116 cells for further functional analysis.

Silencing of RTKN2 inhibits cell growth and induces G1 cell cycle arrest in SW480 and HCT116 cell lines. To investigate the role of RTKN2 on the proliferation of human colon cancer cells, the proliferation of RTKN2 silencing cells was examined using a CCK-8 assay. As shown in Fig. 2D and E, cell growth was significantly suppressed in RTKN2 shRNA virus-infected cells (SiRTKN2) compared with scramble shRNA virus-infected cells (SiNC) and wild-type cells (Con) in SW480 and HCT116 cells. These results suggested that RTKN2 promoted colon cancer cells proliferation.

The present study then examined the possible inhibitory effect of RTKN2 silencing on cell cycle progression. As presented in Fig. 2F-I, cell cycle analysis suggested that the population of RTKN2 knockdown in SW480 and HCT116 cells in G0/G1 phase was markedly increased by 20 and $41.1 \%$, respectively $(\mathrm{P}<0.05)$, and the population of $\mathrm{S}$-phase cells was significantly reduced, by 33.3 and $43.8 \%$, respectively $(\mathrm{P}<0.05)$, compared with scramble shRNA SiNC cells. The data indicated a role for RTKN2 in the promotion of colon cancer G1/S cell cycle transition.

Suppression of RTKN2 expression induces apoptosis of SW480 and HCT116 cells. To determine whether RTKN2 affected the apoptosis ability of the human colon cancer cells, Annexin V/PI staning and flow cytometric analysis were performed. The ratio of cells undergoing apoptosis was markedly increased, by $21.1 \pm 0.9 \%$ in the RTKN2 shRNA virus-infected SW480 cells, compared with SiNC (2.7\%) and Con (2.3\%) (Fig. 3A). Similar results were observed in HCT116 cells. HCT116 cells were increased by $29 \pm 1.2 \%$ in the SiRTKN2 group, compared with the SiNC group (2.1\%) and Con group (2.5\%) (Fig. 3B). These data obtained in the present study indicated that RTKN2 may play an important anti-apoptosis role in human colon cancer cells. 
A

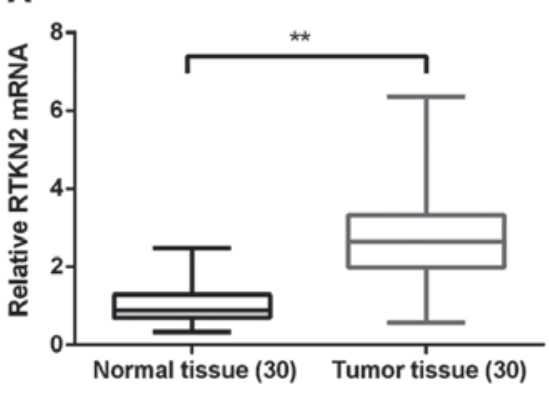

D

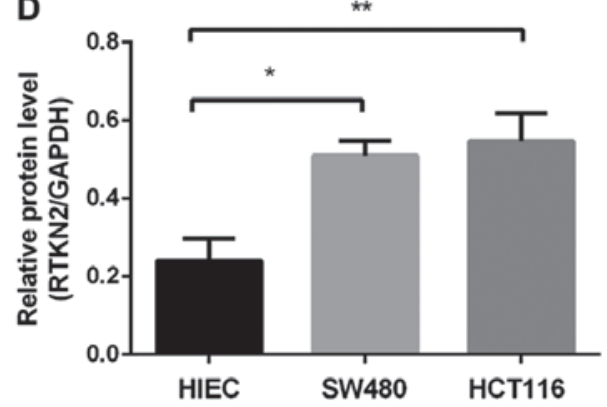

$\mathbf{F}$

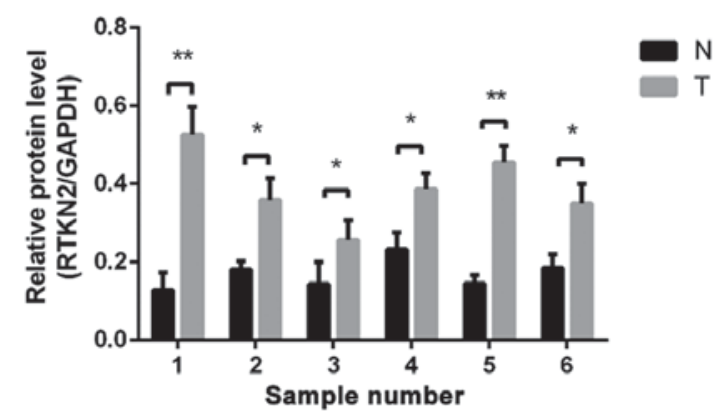

B

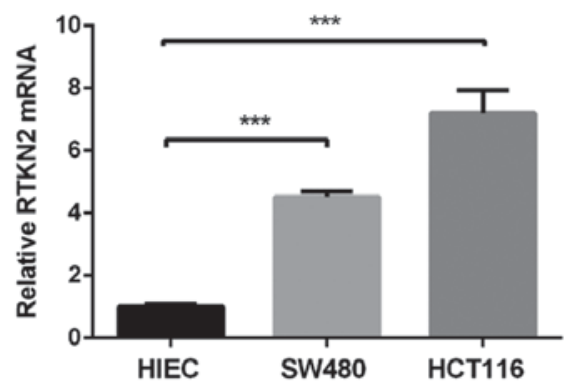

E

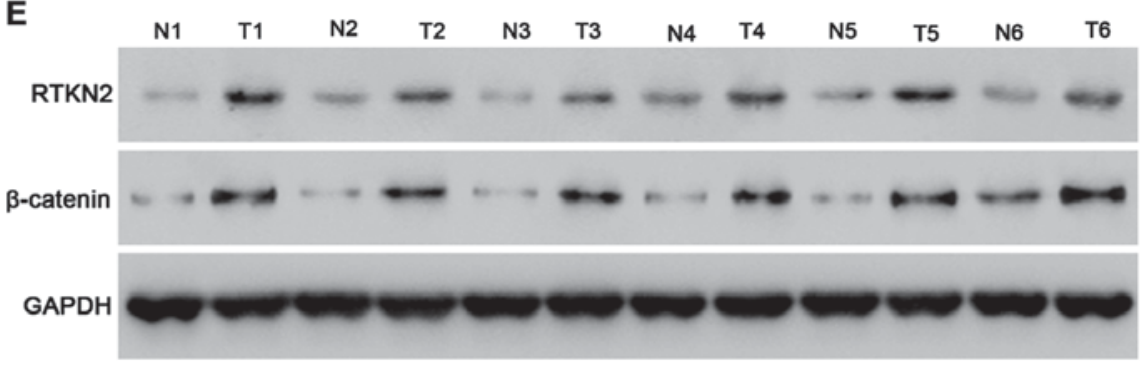

C

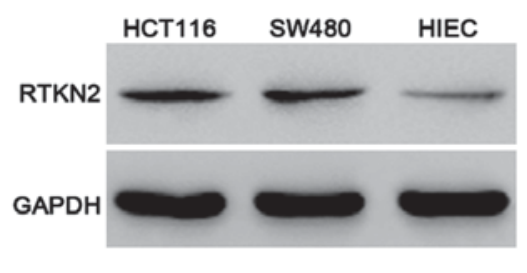

G

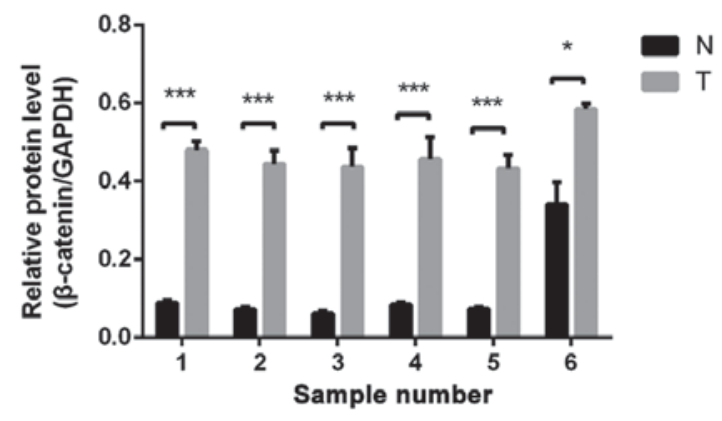

Figure 1. RTKN2 is upregulated in human colon cancer tissues and cell lines. (A) The mRNA expression level of RTKN2 in colon cancer tissues and paired non-cancerous tissues. (B) The mRNA levels of RTKN2 in SW480, HCT116 and HIEC cells. (C and E) Protein levels of RTKN2 in SW480, HCT116 and HIEC cells. (D, F and G) Protein levels of RTKN2 and $\beta$-catenin in colon cancer (T1-T6) and non-cancerous tissues $(\mathrm{N} 1-\mathrm{N} 6)$. ${ }^{*} \mathrm{P}<0.05,{ }^{* * *} \mathrm{P}<0.01,{ }^{* * * *} \mathrm{P}<0.001 . \mathrm{RTKN} 2$, Rhotekin 2; GAPDH, glyceraldehyde 3-phosphate dehydrogenase.

Silencing of RTKN2 inhibits the expression levels of cell apoptosis-associated proteins and anti-apoptosis protein. To investigate the possible mechanism of RTKN2 knockdown that inhibits colon cell proliferation, induces G1 cell cycle arrest and apoptosis, western blot analysis was performed to detect PCNA, cell cycle-associated proteins and cell apoptosis-associated proteins in SW480 and HCT116 cells. RTKN2 silencing resulted in marked downregulation in the levels of PCNA, Cyclin D1 and c-myc in the SW480 and HCT116 cells, compared with the SiNC group of cells (Fig. 4A-D). These results showed that RTKN2 knockdown suppressed the expression of PCNA and cell cycle-associated proteins, which may have contributed to the inhibition of cell proliferation and induction of G1 phase cell cycle arrest. In addition, it was found that RTKN2 silencing inhibited the expression of $\mathrm{Wnt} / \beta$-catenin protein pathway. To prove that RTKN2 silencing inhibited colon cancer cell proliferation by inhibiting the activity of Wnt/ $\beta$-catenin pathway, HCT116 cells were treated with $\mathrm{Wnt} / \beta$-catenin pathway agonist LiCI and RTKN2 was silenced. The results showed that RTKN2 silencing inhibited the activation effect of $\mathrm{LiCI}$ on the
Wnt/ $\beta$-catenin pathway and decreased the expressions of cell cycle-associated proteins induced by LiCI (Fig. 4E and F), thereby inhibiting cell proliferation.

In addition, the data indicated the silencing of RTKN2 inhibited the expression of anti-apoptosis protein $\mathrm{Bcl} 2$ and increased the expression of pro-apoptosis protein Bax (Fig. 4A and B), which resulted in cell apoptosis.

\section{Discussion}

RTKN belongs to the group of proteins containing a Rho-binding domain, which are target effectors for Rho-GTPases (4). The RTKN gene was only identified recently and its physiological functions remain largely unknown (12). RTKN has been reported to be associated with several cancer types including HCC (5), breast cancer (10), gastric cancer (13), and bladder cancer (11). However, the molecular mechanisms underlying the development and progression of human colon cancer have yet to be reported. In the present study, RT-qPCR and western blot analysis showed that the expression levels of RTKN2 mRNA and protein were markedly higher in human 
A

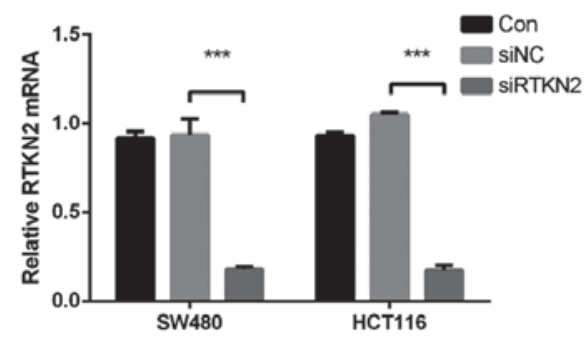

D

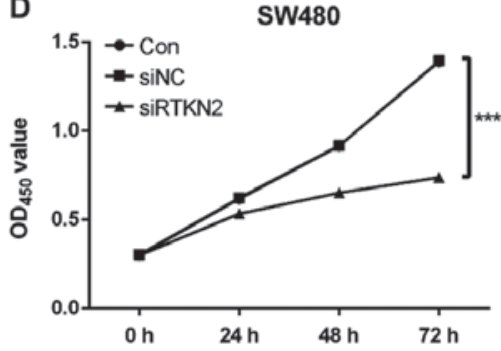

G

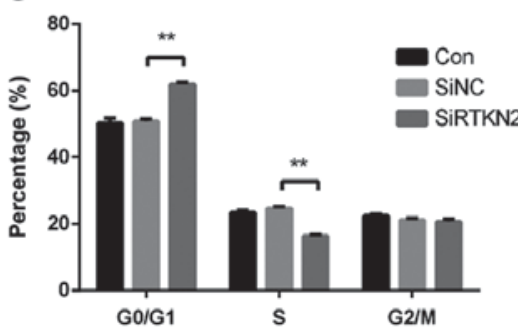

B

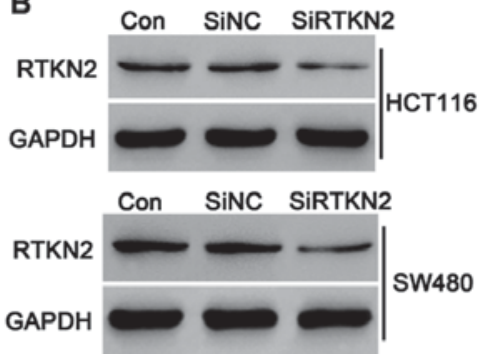

E

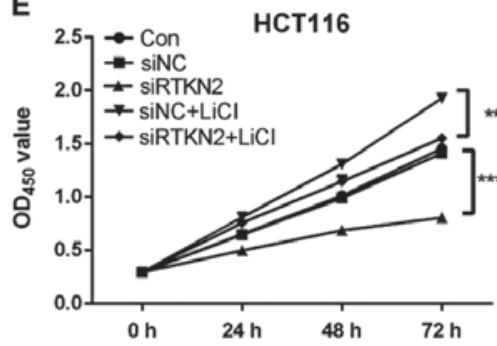

H

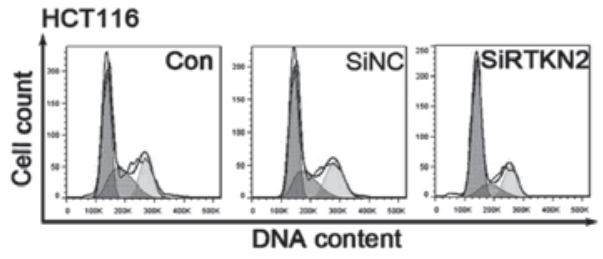

c

F
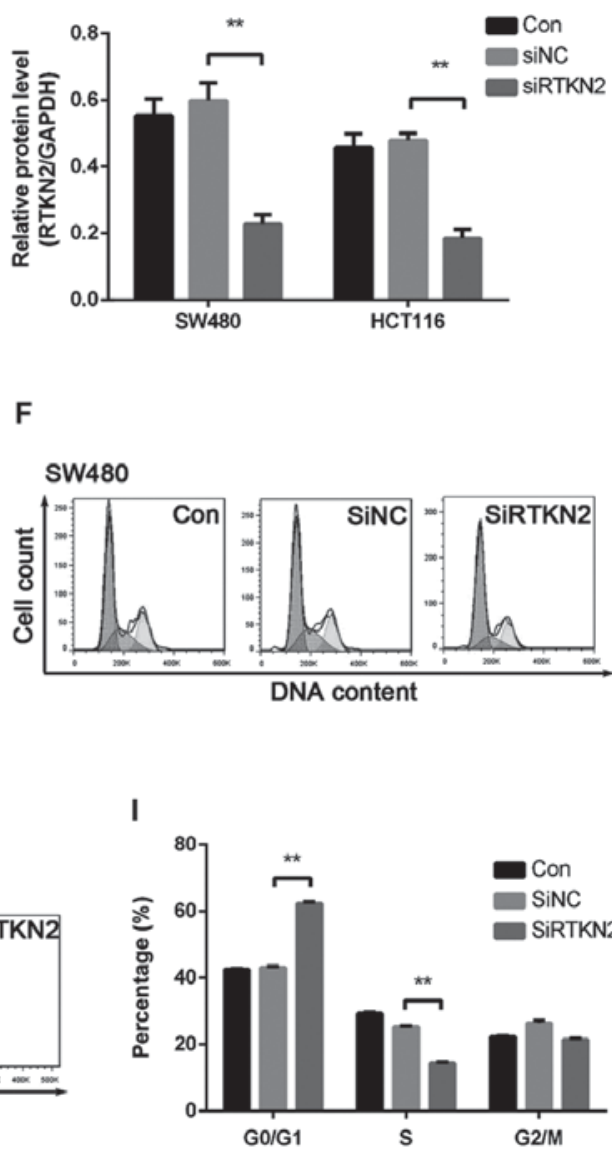

Figure 2. Silencing of RTKN2 inhibits cell growth and induces G1 cell cycle arrest in SW480 and HCT116 cell lines. (A) Knockdown of RTKN2 expression in HCT116 and SW480 cells as indicated by RT-PCR analysis. (B and C) Knockdown of RTKN2 expression in HCT116 and SW480 cells as indicated by western blot analysis. (D and E) Cell proliferation was detected at 24, 48 and $72 \mathrm{~h}$ after shRNA virus infection in SW480 (D) and HCT116 cells (E). (F and I) Cell cycle progression was analyzed using flow cytometry in RTKN2 knockdown cells in SW480 (F and G) and HCT116 cells (H and I). ${ }^{* *} \mathrm{P}<0.01$, ${ }^{* * * *} \mathrm{P}<0.001$. RTKN2, Rhotekin 2; GAPDH, glyceraldehyde 3-phosphate dehydrogenase.

colon cancer tissues, compared with adjacent normal tissues. In addition, the data showed RTKN2 was expressed at high levels in SW480 and HCT116 colon cancer cells.

Cell cycle progression is frequently abnormal in the majority of cancer types, leading to aberrant cell growth $(14,15)$. In the present study, the effects of RTKN2 on proliferation, cell cycle progression and apoptosis were examined by silencing its expression in SW480 and HCT116 cells. It was observed that the suppression of RTKN2 markedly inhibited cell proliferation. Furthermore, flow cytometric analysis revealed that knockdown of RTKN notably induced G1 phase cell cycle arrest in SW480 and HCT116 cells, which suggested that the suppression of cell growth in colon cancer cells was due to the arrest of cell cycle progression. The expression of the cell cycle regulators, PCNA, Cyclin D1 and c-myc, was additionally confirmed. The results of the present study suggested that knockdown of RTKN2 inhibited the expression levels of PCNA, Cyclin D1 and c-myc in SW480 and HCT116 cells, which was consistent with the results of cell growth and the cell cycle analysis.

Wnt signaling pathways can be divided into classical (Wnt/ $\beta$-catenin signaling) and non-classical (Wnt/PCP signaling). The classical typical pathway, Wnt/ $\beta$-catenin signaling pathway, plays an important role in tumor cell proliferation, differentiation, metastasis and invasion (16-18). The changes in the transcriptional activity and protein stability of $\beta$-catenin are important indexes of $\mathrm{Wnt} / \beta$-catenin signaling pathway activation. The results of our research showed that Wnt/ $\beta$-catenin pathway was activated in colon cancer and RTKN2 silencing inhibited the $\beta$-catenin expression. Cyclin D1 is a key regulatory protein in the G1 phase and a downstream target gene of $\mathrm{Wnt} / \beta$-catenin pathway. To confirm whether there was any inhibitory effect of RTKN2 on colon cancer cell proliferation following inhibition of the Wnt/ $\beta$-catenin signaling pathway, HCT116 cells were treated with Wnt/ $\beta$-catenin pathway agonist LiCI and RTKN2 expression was silenced in this study. The results revealed that RTKN2 silencing reduced the activation effect of $\mathrm{LiCl}$ on the Wnt/ $\beta$-catenin signaling pathway and reduced the expression of Cyclin D1 and c-myc induced by LiCI. The CCK-8 results showed that RTKN2 silencing reduced the promoting effect of LiCI on colon cancer cell proliferation in HCT116 cells. These results suggested that TRKN2 silencing inhibits colon cancer cell proliferation by inhibiting the Wnt/ $\beta$-catenin signaling pathway.

Previous studies have confirmed the anti-apoptotic effects of RTKN2 on several cancer types including HCC and bladder cancer $(5,6)$. Consistent with these observations, the results 
A

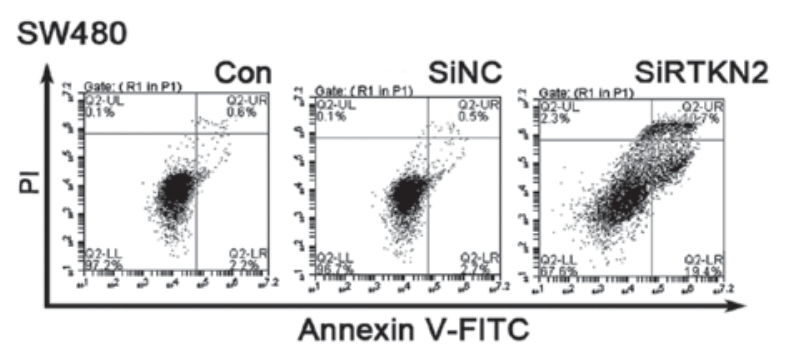

B

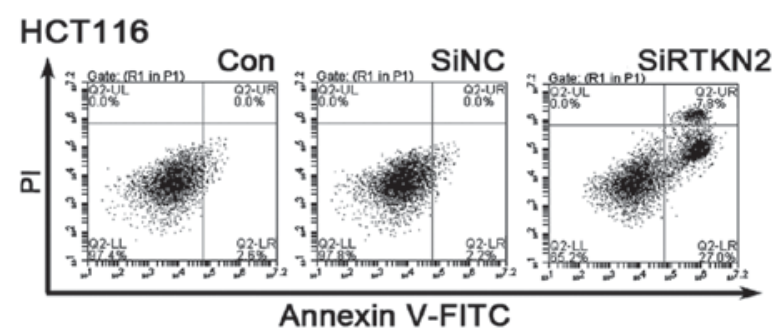

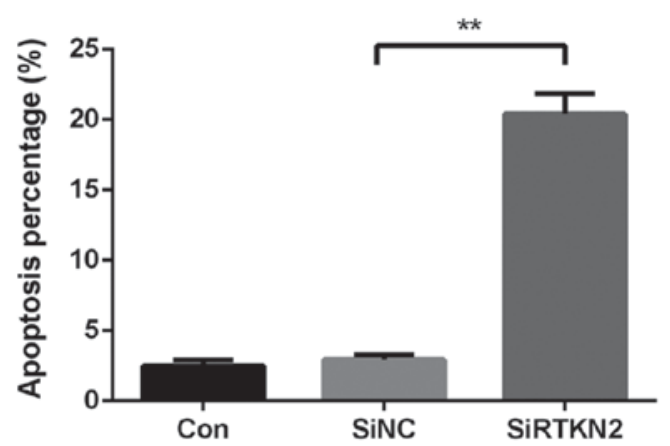

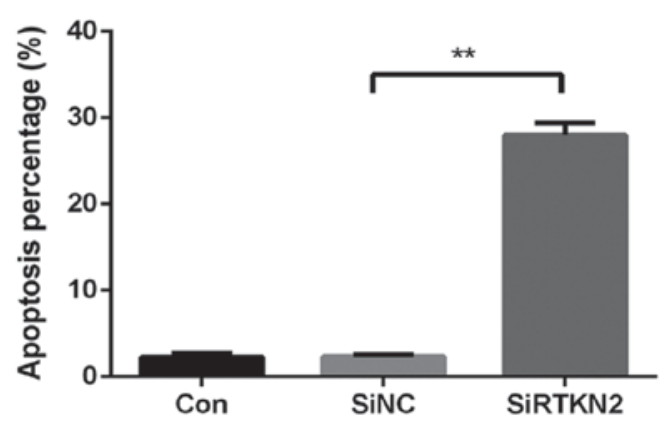

Figure 3. Suppression of RTKN2 expression induced apoptosis of SW480 and HCT116 cells. SW480 (A) and HCT116 cells (B) were overexpressed with RTKN2, stained with Annexin V/PI and apoptosis percentages were examined using flow cytometry. " $\mathrm{P}<0.01$. PI, propidium iodide solution.

A

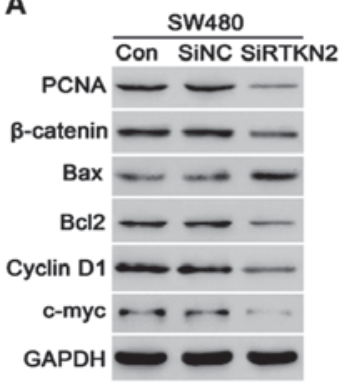

B

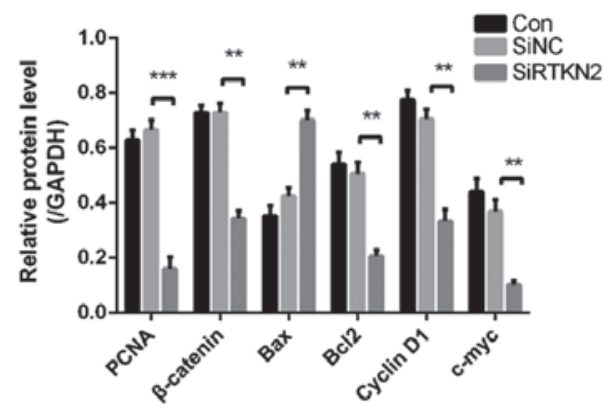

C

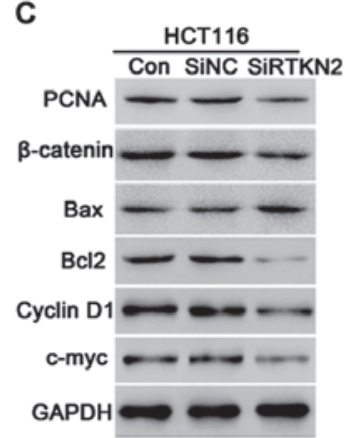

D

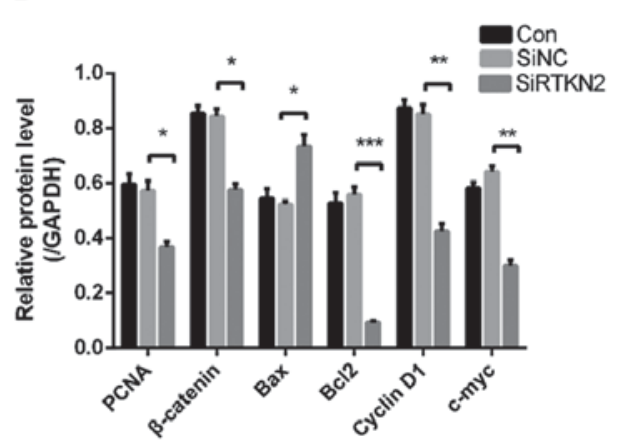

E

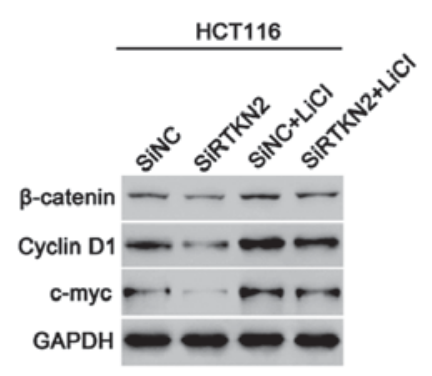

$\mathrm{F}$

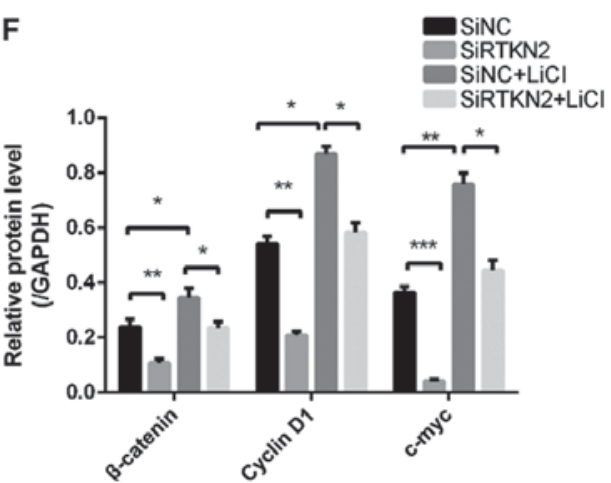

Figure 4. Silencing of RTKN2 inhibits the expression levels of cell cycle-associated proteins and anti-apoptosis protein. (A and D) The protein levels of PCNA, $\beta$-catenin, Cyclin D1, c-myc, Bax and Bcl2 were altered in SW480 (A and B) and HCT116 cells (C and D) after RTKN2 knockdown. (E and F) HCT116 cells were treated with Wnt/ $\beta$-catenin pathway agonist LiCI and RTKN2 was silenced. The protein levels of $\beta$-catenin, Cyclin D1 and c-myc were measured by western blot analysis. ${ }^{*} \mathrm{P}<0.05,{ }^{* *} \mathrm{P}<0.01,{ }^{* * *} \mathrm{P}<0.001$. GAPDH, glyceraldehyde 3-phosphate dehydrogenase.

of the present study demonstrated that silencing of RTKN2 markedly induced the apoptosis of human colon cancer cells.

Taken together, the present results indicated that RTKN2 was upregulated in human colon cancer tissues and cells. Knockdown of RTKN2 suppressed cell proliferation and induced cell apoptosis. Furthermore, suppression of RTKN2 expression induced the G1 phase arrest. In addition, the Wnt/ $\beta$-catenin signaling pathway was activated in human colon cancer tissues, and 
silencing of RTKN2 suppressed colon cell proliferation through inhibition of the activation of Wnt/ $\beta$-catenin. However, whether RTKN2 can be used as a diagnostic marker or therapeutic target for colon cancer remains to be further investigated.

\section{Acknowledgements}

This work was funded by the National Natural Science Foundation of China (81401943) and Suzhou science and technology program (SYS201462).

\section{References}

1. Jemal A, Bray F, Center MM, Ferlay J, Ward E and Forman D, et al: Global cancer statistics. CA Cancer J Clin 61: 69-90, 2011.

2. Wilkes GM: Therapeutic options in the management of colon cancer: 2005 update. Clin J Oncol Nurs 9: 31-44, 2005.

3. Winawer S, Fletcher R, Rex D, Bond J, Burt R, Ferrucci J, Ganiats T, Levin T, Woolf S, Johnson D, et al: Gastrointestinal consortium panel: Colorectal cancer screening and surveillance: Clinical guidelines and rationale-Update based on new evidence. Gastroenterology 124: 544-560, 2003.

4. Collier FM, Gregorio-King CC, Gough TJ, Talbot CD, Walder K and Kirkland MA: Identification and characterization of a lymphocytic Rho-GTPase effector: Rhotekin-2. Biochem Biophys Res Commun 324: 1360-1369, 2004.

5. Wei W, Chen H and Liu S: Knockdown of Rhotekin 2 expression suppresses proliferation and invasion and induces apoptosis in hepatocellular carcinoma cells. Mol Med Rep 13: 4865-4871 2016

6. Liao YX, Zeng JM, Zhou JJ, Yang GH, Ding K and Zhang XJ: Silencing of RTKN2 by siRNA suppresses proliferation, and induces G1 arrest and apoptosis in human bladder cancer cells. Mol Med Rep 13: 4872-4878, 2016.

7. Gregorio-King CC, Gough T, Van Der Meer GJ, Hosking JB, Waugh CM, McLeod JL, Collier FM and Kirkland MA: Mechanisms of resistance to the cytotoxic effects of oxysterols in human leukemic cells. J Steroid Biochem Mol Biol 88: 311-320, 2004
8. Aupeix K, Weltin D, Mejia JE, Christ M, Marchal J,Freyssinet JM and Bischoff P: Oxysterol-induced apoptosis in human monocytic cell lines. Immunobiology 194: 415-428, 1995.

9. Tang J, Chen JX, Chen L, Tang JY, Cui Z, Liu CH and Wang Z: Metastasis associated in colon cancer 1 (MACC1) promotes growth and metastasis processes of colon cancer cells. Eur Rey Med Pharmacol Sci 20: 2825-2834, 2016.

10. Chen M, Bresnick AR and O'Connor KL: Coupling S100A4 to Rhotekin alters Rho signaling output in breast cancer cells. Oncogene 32: 3754-3764, 2013.

11. Fan J, Ma LJ, Xia SJ, Yu L, Fu Q, Wu CQ, Huang XH, Jiang JM and Tang XD: Association between clinical characteristics and expression abundance of RTKN gene in human bladder carcinoma tissues from Chinese patients. J Cancer Res Clin Oncol 131: 157-162, 2005.

12. Fu Q, Yu L, Liu Q, Zhang J, Zhang H and Zhao S: Molecular cloning, expression characterization, and mapping of a novel putative inhibitor of rho GTPase activity, RTKN, to D2S145-D2S286. Genomics 66: 328-332, 2000.

13. Yildiz B, Etiz D, Dal P, Junushova B, Pasaoglu O, Yilmaz E, Erkasap S and Dincer M: Tumor deposits: Prognostic significance in gastric cancer patients. J BUON 21: 1476-1481, 2016.

14. Wang S, Bian C, Yang Z, Bo Y, Li J, Zeng L, Zhou H and Zhao RC: miR-145 inhibits breast cancer cell growth through RTKN. Int J Oncol 34: 1461-1466, 2009.

15. Sevli S, Uzumcu A, Solak M, Ittmann M and Ozen M: The function of microRNAs, small but potent molecules, in human prostate cancer. Prostate Cancer Prostatic Dis 13: 208-217, 2010.

16. Shi L, Wu YX, Yu JH, Chen X, Luo XJ and Yin YR: Research of the relationship between $\beta$-catenin and c-myc-mediated Wnt pathway and laterally spreading tumors occurrence. Eur Rev Med Pharmacol Sci 21: 252-257, 2017.

17. Hua F, Liu S, Zhu L, Ma N, Jiang S and Yang J: Highly expressed long non-coding RNA NNT-AS1 promotes cell proliferation and invasion through $\mathrm{Wnt} / \beta$-catenin signaling pathway in cervical cancer. Biomed Pharmacother 92: 1128-1134, 2017.

18. Zhang Q, Lou Y, Bai X and Liang T: EPAS1 promote tumor progression by interacting with $\mathrm{Wnt} / \beta$-catenin signaling in pancreatic cancer. HPB 18: e350-e351, 2016. 